\title{
Scale dependence of galaxy biasing investigated by weak gravitational lensing: An assessment using semi-analytic galaxies and simulated lensing data (Corrigendum)
}

\author{
Patrick Simon ${ }^{1}$ and Stefan Hilbert ${ }^{2,3}$
}

\footnotetext{
1 Argelander-Institut für Astronomie, Universität Bonn, Auf dem Hügel 71, 53121 Bonn, Germany e-mail: psimon@astro.uni-bonn.de

2 Faculty of Physics, Ludwig-Maximilians University, Scheinerstr. 1, 81679 München, Germany

${ }^{3}$ Excellence Cluster Universe, Boltzmannstr. 2, 85748 Garching, Germany
}

A\&A 613, A15 (2018), https://doi.org/10.1051/0004-6361/201732248

Key words. gravitational lensing: weak - large-scale structure of Universe - cosmology: observations - galaxies: statistics errata, addenda

We correct typographical errors in four equations of the original paper. These errors have no impact on our results and conclusions because the correct equations were implemented in the codes used for the original analysis.

A detailed list of the typographical errors follows. There is a minus sign missing in the approximation of Eq. (31). The correct approximation is

$$
\begin{aligned}
F_{\mathrm{ia}}(\chi) & :=-A_{\mathrm{ia}} \mathrm{C}_{1} \bar{\rho}_{\text {crit }} \frac{\Omega_{\mathrm{m}}}{D_{+}(\chi)} \\
& \approx-2.4 \times 10^{-2}\left(\frac{A_{\mathrm{ia}}}{3.0}\right)\left(\frac{\Omega_{\mathrm{m}}}{0.3}\right)\left(\frac{D_{+}(\chi)}{0.5}\right)^{-1} .
\end{aligned}
$$

The exponent of the matter density parameter, $\Omega_{\mathrm{m}}$, in Eq. (34) has to be 2,

$P_{\mathrm{n} \kappa}^{(2)}(\ell)=-\frac{9 H_{0}^{4} \Omega_{\mathrm{m}}^{2}}{2 c^{4}} \int_{0}^{\chi \mathrm{h}} \mathrm{d} \chi \frac{g_{\mathrm{s}}(\chi) g_{\mathrm{d}}(\chi)}{a^{2}(\chi)} P_{\mathrm{m}}\left(k_{\ell}^{\chi} ; \chi\right)$.

The pre-factor of the first integral in Eq. (36) has an incorrect exponent for the inverse Hubble length, $H_{0} c^{-1}$. It should be as follows:

$$
\begin{aligned}
& P_{\mathrm{n}}^{(2)}(\ell ; b, r)=\frac{9 H_{0}^{4} \Omega_{\mathrm{m}}^{2}}{c^{4}} \int_{0}^{\chi_{\mathrm{h}}} \mathrm{d} \chi \frac{g_{\mathrm{d}}^{2}(\chi)}{a^{2}(\chi)} P_{\mathrm{m}}\left(k_{\ell}^{\chi} ; \chi\right) \\
& -\frac{6 H_{0}^{2} \Omega_{\mathrm{m}}}{c^{2}} \int_{0}^{\chi \mathrm{h}} \mathrm{d} \chi \frac{p_{\mathrm{d}}(\chi) g_{\mathrm{d}}(\chi)}{a(\chi) f_{K}(\chi)} b\left(k_{\ell}^{\chi}\right) r\left(k_{\ell}^{\chi}\right) P_{\mathrm{m}}\left(k_{\ell}^{\chi} ; \chi\right) .
\end{aligned}
$$

Equation (60) should have a square for $\langle N \mid m\rangle$,

$$
\langle N(N-1) \mid m\rangle=R^{-2}(k, m) \tilde{u}_{\mathrm{g}}^{2 q-2 p}(k, m)\langle N \mid m\rangle^{2} .
$$

Moreover, contrary to our statement in Sect. 5.2 of the original paper, in the way we set up the formalism, the mean biasing function, $b(m)$, must be normalised to obey Eq. (84), namely

$$
\int_{0}^{\infty} \mathrm{d} m n(m) m b(m)=\int_{0}^{\infty} \mathrm{d} m n(m) m
$$

as correctly stated in Appendix D of Simon \& Hilbert (2021). This was indeed done in our computer codes used for the analysis.

\section{References}

Simon, P., \& Hilbert, S. 2021, A\&A, 646, A71 